\title{
Detección de b-Amiloide, Proteína TAU Hiperfosforilada y Ubiquitina por Técnica de Inmunohistoquímica en Cerebros de Caninos Mayores de 10 Años
}

\author{
Detection b-Amiloid, Hyperphosphorylate TAU Protein and Ubiquitin for \\ Technique the Immunohistochemistry in Brains in Dogs over 10 Years
}

"Flavio Briones S.; **Tamara Cáceres D. \& ** Marcia Jarpa F.

BRIONES, S. F.; CÁCERES, D. T. \& JARPA, F. M. Detección de b-Amiloide, proteína TAU hiperfosforilada y ubiquitina por técnica de inmunohistoquímica en cerebros de caninos mayores de 10 años. Int. J. Morphol., 28(4):1255-1261, 2010.

RESUMEN: El interés en el estudio del proceso de envejecimiento cerebral y los cambios comportamentales relacionados con la edad en caninos y felinos geriátricos ha aumentado en la última década (Mentzel, 2005a; Ingram \& Williams, 2010). Las alteraciones del comportamiento canino que responden a cambios fisiopatológicos relacionados con la edad y que involucran distintas esferas conductuales y cognitivas, se engloban bajo la denominación de Síndrome de Disfunción Cognoscitiva (SDC) (Mentzel, 2005b). El cual fue descrito en 1997 y se observó en perros de más de 9 años (Ingram \& Williams). El Síndrome de Disfunción Cognoscitivo (SDC) es también llamado Alzheimer canino por las similitudes histopatológicas cerebrales y conductuales relacionadas. En el presente estudio, los caninos geriátricos presentaron los mismos depósitos proteicos anormales presentes en la enfermedad de Alzheimer, como las placas seniles, los cuerpos ubiquitinados y la angiopatía amiloide, pero no presentaron los ovillos neurofibrilares, los cuales son los responsables de la demencia que se padece en la enfermedad. Las únicas herramientas de evaluación prácticas en clínica para esta patología son las encuestas y formularios relacionados con aspectos comportamentales. Además existen exámenes diagnósticos postmortem que permiten identificar de una manera eficaz la presencia de elementos anormales involucrados en la neuropatología, uno de ellos es la inmunohistoquímica. En este estudio se utilizaron los anticuerpos monoclonales de uso humano anti-b-amiloide, anti-proteína tau y antiubiquitina en muestras de cerebros de perros mayores de 10 años. El modelo canino hoy en día está constituyendo un significado indispensable para el estudio de los procesos de neurodegeneración porque ha permitido un acercamiento a la teoría del problema desde nuevas perspectivas (Dimakopoulos \& Mayer, 2002).

PALABRAS CLAVE: Cognoscitiva; Inmunohistoquímica; Amiloide; Proteína TAU; Ubiquitina.

\section{INTRODUCCIÓN}

El cuidado hacia las mascotas ha aumentado significativamente en los últimos años, prolongando su expectativa de vida. Esto a su vez ha llevado a la presentación de nuevas patologías relacionadas con la edad. Dentro de estas patologías, las que más llaman la atención son las que involucran cambios comportamentales, ya que influyen directamente en la relación mascota dueño.

Los propietarios de perros geriátricos con problemas de comportamiento suelen explicarlos al Médico Ve- terinario para que intente orientarles, pero éste suele tener un conocimiento limitado de los mecanismos subyacentes y dispone de muy pocas herramientas terapéuticas a su alcance (Ingram \& Williams, 2010). Es por ello que muchos dueños finalmente aceptan que los cambios asociados al comportamiento adverso en sus perros son una consecuencia inevitable del proceso de envejecimiento (Platt, 2005).

El perro geriátrico presenta un equivalente a la enfermedad de Alzheimer humana, llamado Síndrome de

\footnotetext{
* Magister, Médico Veterinario, Universidad Iberoamericana de Ciencias y Tecnología, Santiago, Chile.

** Médico veterinario, Universidad Iberoamericana de Ciencias y Tecnología, Santiago, Chile.

Esta investigación fue parte del proyecto A-018-0312 "Detección de b-amiloide, ubiquitina y ovillos neurofibrilares en cerebros de caninos geriátricos mediante inmunohistoquímica”, fue financiado con fondos de investigación de la Universidad iberoamericana de Ciencias y Tecnología.
} 
Disfunción Cognoscitiva, el cual fue descrito por Landesberg \& Ruehl en 1997 y se observó en perros mayores de 9 años (Ingram \& Williams), el cual es causado por cambios químicos y físicos en el cerebro no atribuibles sólo a un envejecimiento normal (Godoy, 2004). En Santiago de Chile, la prevalencia en perros mayores de 9 años es $27.4 \%$; en los mayores de 15 años, alcanza el $61,1 \%$ (Briones, 2006).

Tanto en la enfermedad de Alzheimer humana como su similar en los caninos, se presentan depósitos proteicos anormales que corresponden a placas seniles y ovillos neurofibrilares. Su presencia permite llegar a un diagnóstico definitivo, sin embargo no son exclusivas de ella, ya que pueden estar presentes en otras patologías.

Las placas seniles son lesiones del neurópilo, de estructura esferoide que se encuentran en el espacio extraneuronal y están constituidas principalmente por una proteína llamada b-amiloide (Guimerà et al., 2002). bamiloide, es un péptido de longitud variable de 39 a 43 aminoácidos y de un tamaño de 4-6 kDa. Esta proteína es un producto natural del metabolismo de la proteína precursora del amiloide, la cual posee características estructurales de una proteína de membrana (Gra et al., 2002).

En perros al igual que en humanos las paredes de los vasos sanguíneos son otro punto de acumulación de bamiloide (Mentzel, 2005a), este fenómeno se denomina Angiopatía Amiloide. La angiopatía amiloide cerebral es una neuropatología característica de la enfermedad de Alzheimer, la cual se caracteriza por depósitos de bamiloide principalmente en las paredes de los vasos sanguíneos corticales y de las leptomenínges (Prior et al., 1996; Weigel et al., 1995).

Los ovillos neurofibrilares están constituidos principalmente por una proteína designada como tau. La proteína tau en las neuronas es la principal representante de las proteínas asociadas a los microtúbulos llamadas habitualmente MAP (Microtubule-Associated Proteins) (Morelli \& Castaño, 2004). Los microtúbulos, son uno de los 3 constituyentes mayores del citoesqueleto neuronal. En las neuronas los microtúbulos participan en la formación de las prolongaciones típicas de estas células (axón y dendritas), en el transporte de nutrientes y en el mantenimiento de la estabilidad estructural de la neurona (Gra $e t$ al.; Morelli \& Castaño).

La estabilidad estructural de los microtúbulos del citoesqueleto neuronal depende entre otros factores de la eficiencia de la unión de tau a ellos. Esta unión a su vez es modulada por la asociación de fosfato a tau (Morelli \&
Castaño). Cuando esta proteína se une a más grupos fosfatos de lo normal, se presenta su forma anormal llamada Proteína Tau Hiperfosforilada, la cual conduce a un mal ensamble y desensamblaje alterado de los microtúbulos y también contribuye a una incorporación adicional de tau normal en filamentos anormales (Guimerà et al.). Este proceso lleva a la formación de los Ovillos Neurofibrilares, los cuales son los responsables de la demencia que se padece en la enfermedad de Alzheimer.

Hace algunos años se descubrió un tercer depósito, los Cuerpos Ubiquitinados, los cuales están constituidos por una pequeña proteína llamada ubiquitina, cuya función principal es "marcar" proteínas anómalas o envejecidas para su posterior degradación por una estructura denominada proteasoma. La ubiquitina es una pequeña proteína compuesta por 76 aminoácidos, la cual se pliega en una estructura globular y compacta. Llamada así por ser muy abundante y estar en todas partes (ubicua). Esta proteína sólo es encontrada en organismos eucariontes, en donde se encuentra en forma libre o unida covalentemente a otras proteínas (Alberts et al., 1996; Briones et al.; Lozano, 1995). Al igual que la proteína tau se encuentra dentro de la célula nerviosa.

\section{MATERIAL Y METODO}

El estudio se llevo a cabo en el Laboratorio de Histopatología de la Universidad Iberoamericana de Ciencias y Tecnología. Se utilizaron 43 muestras de cerebros caninos mayores de 10 años sin restricción de sexo, raza y estado de salud. Se les aplicó la técnica de inmunohistoquímica de inmunoperoxidasa con método indirecto. Para realizar la técnica de inmunohistoquímica se utilizaron los siguientes reactivos: Anticuerpos primarios monoclonales (Novocastra Laboratories Ltd) Ubiquitin lyophilised monoclonal antibody, clone FPM1, concentrado-1 ml; Beta Amyloid and Tau (duo pack) lyophilised monoclonal, clone 6F/3D and Tau-2. Anticuerpo secundario: Biotinylated Anti-mouse IgG $(\mathrm{H}+\mathrm{L})$, made in horse, 1,5 mg (E-0413) (Vector laboratories-U.S.A). Enzima: Horseradish Peroxidase Streptavidin- $1 \mathrm{mg}$ (P-0397) (Vector Laboratories- U.S.A). Cromógeno: 3,3'Diaminobenzidine tetrahydrochloride Substrate Kit (DAB). Histochemical. Substrate for horseradish peroxidase (Zymed Laboratories INC).

Los controles positivos utilizados para estandarizar la técnica de inmunohistoquímica fueron de corteza temporal de un paciente humano que falleció con la enfermedad de Alzheimer, la muestra presentaba las lesiones 
histopatológicas características de la enfermedad (placas seniles, ovillos neurofibrilares y cuerpos ubiquitinados), la cual fue diagnosticada tanto clínicamente como histopatológicamente mediante diversas técnicas de laboratorio. Adicionalmente para la estandarización de la técnica para el anticuerpo antiubiquitina se utilizaron controles positivos de biopsias hepáticas, las cuales fueron utilizadas para el diagnóstico de hepatitis alcohólica, donde se encuentra la presencia de los denominados Cuerpos de Mallory (CM) que presentaron inmunoreactividad a ubiquitina.

Se utilizaron cortes de 8 micras de grosor, de corteza frontal y temporal para estudiar los depósitos de b-amiloide ya que son áreas directamente involucradas con la cognición. Para el anticuerpo anti-ubiquitina y anti-tau no se utilizó corteza frontal ya que por antecedentes bibliográficos, los depósitos de proteína tau pueden observarse en regiones cerebrales distintas a las afectadas por los depósitos de bamiloide. Además los controles humanos utilizados para estandarizar la técnica de inmunohistoquímica fueron de corteza temporal por ser una zona tributaria de la enfermedad de Alzheimer. Es por esa razón que se utilizó esa zona, ya que si este estudio propone al perro como un futuro modelo para el estudio de la enfermedad de Alzheimer, se debía estudiar los diversos depósitos proteicos tanto humanos como caninos en la misma zona.

Para llevar a cabo el análisis de los resultados previamente se fotografiaron con una cámara digital (Nikon 4200) 10 fotos por cada anticuerpo utilizado en las áreas seleccionadas anteriormente (Tabla I).

Una vez obtenidas las fotografías se procedió a contabilizar los elementos en las determinadas áreas. Para ello se utilizó el programa Image Tool versión 3.00. Para cada anticuerpo se contabilizaron los elementos descritos en la Tabla II. Para el caso del b-amiloide además de placas se contabilizaron vasos sanguíneos leptomeníngeos positivos al anticuerpo. Para ello se seleccionaron 3 áreas en donde se contabilizaron tanto vasos negativos como positivos. Posteriormente se calculó el área correspondiente a un campo microscópico para cada fotografía y se estandarizó al equivalente de una superficie de $500.000 \mathrm{~m}^{2}$, de esa manera se pudo homogeneizar el área para los 3 anticuerpos utilizados y extrapolar los elementos positivos en dicha área.

Para pesquisar los cambios comportamentales y el grado de disfunción cognoscitivo que se presenta en esta patología se utilizó un Sistema de Evaluación para el Diagnóstico del Síndrome de Disfunción Cognoscitivo Canino, el cual consiste en una encuesta que se le realiza al dueño de las mascotas comprometidas y evalúa los principales comportamientos que se ven alterados en este cuadro, dividiendo la evaluación en 5 categorías que incluyen : Orientación, interacción con los miembros de la familia, hábitos de eliminación (micción y defecación) y el ciclo sueño vigilia.

Algunos de los signos presentes en los pacientes con síndrome de disfunción cognoscitivo fueron los siguientes: el perro no reconoce a su familia, no atiende a ordenes o por su nombre, camina sin sentido, duerme menos por la noche adoptando conductas que antes no realizaba (ladrar, caminar o vociferar), duerme mas durante el día, orina o defeca donde antes no lo hacia, solicita menos atención, no quiere recibir caricias, no saluda a los dueños una vez que se entera que han llegado, entre otros. La encuesta utilizada es una adaptación de Briones y Marcos (2) de la original, diseñada por el Doctor David Hammet (2003), Médico Veterinario de la clínica All Creatures (Tennessee).

Para el análisis estadístico de las diversas variables de este estudio se utilizó el test estadístico de Kruskal - Wallis y luego el test de comparación múltiple de Dunn. Cabe mencionar que para llevar a cabo los diferentes análisis, la muestra total se dividió en 3 grupos etáreos: el primero entre 10 a 12 años, el segundo entre 13 a 15 años y por último los mayores de 15 años.

Tabla II. Elementos contabilizados y patrones de tinción para cada anticuerpo.

\begin{tabular}{lll}
\hline Anticuerpo & Elementos contabilizados & Patrón de tinción \\
\hline -amiloide & Placas seniles & Extracelular \\
Proteína Tau & Células de la glia positivas & Intracelular \\
Ubiquitina & Gránulos y puntillado & Intracelular \\
\hline
\end{tabular}

Tabla I. Áreas fotografiadas para cada anticuerpo.

\begin{tabular}{lll}
\hline Anticuerpo & Areas fotografiadas & Aumento \\
\hline --amiloide & Sustancia gris de corteza frontal (capas profundas) & $100 \mathrm{X}$ \\
Proteína Tau & Sustancia blanca de corteza temporal & $400 \mathrm{X}$ \\
Ubiquitina & Sustancia blanca de corteza temporal & $400 \mathrm{X}$ \\
\hline
\end{tabular}




\section{RESULTADOS}

En el estudio los caninos geriátricos presentaron los mismos depósitos proteicos anormales presentes en la enfermedad de Alzheimer, como las placas seniles, los cuerpos ubiquitinados y la angiopatía amiloide, pero no presentaron los ovillos neurofibrilares.

La mayoría de los caninos (70\%) presentes en el estudio mostraron signos de disfunción al momento de realizar la encuesta. Los individuos que presentaron mayor prevalencia al síndrome de disfunción son los mayores a 15 años (88\%). Pese a esto no hubo correlación estadísticamente significativa, sólo una tendencia a aumentar los signos a medida que se incrementó la edad.

Las placas de b-amiloide resultaron ser positivas en el $86 \%(n=37)$ de las muestras. Estas se depositaron en la sustancia gris de la corteza frontal, principalmente en las capas profundas (Fig. 1).
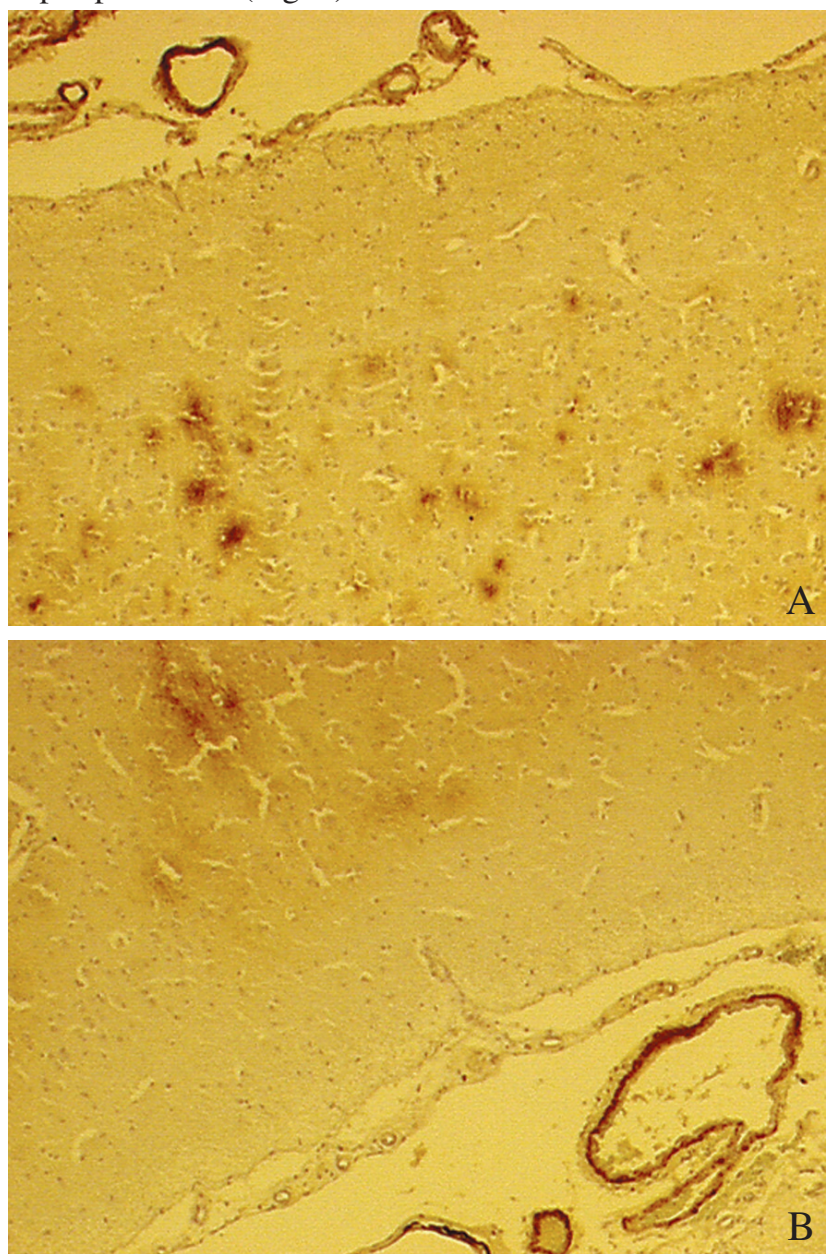

Fig. 1. Presencia de ambas lesiones amiloideas en corteza frontal de cerebro canino (400X).
Del total de las muestras un 67\% $(\mathrm{n}=29)$ presentaron vasos sanguíneos leptomeníngeos positivos a b-amiloide (Fig. 2). Del total de las muestras un $76 \%(\mathrm{n}=28)$ presentaron ambas lesiones amiloideas (placas seniles y angiopatía amiloide). Según el análisis estadístico no hubo correlación estadísticamente significativa. Esta misma situación se dio en humanos.

El promedio de placas seniles por grupos etáreos, según el análisis estadístico no mostró correlación estadísticamente significativa. Sin embargo, perros mayores de 15 años fueron los que mayor número de placas presentaron. No se observó correlación entre el número de placas seniles y la disfunción cognoscitiva. Pese a esto las placas se encuentran en mayor proporción en individuos que resultaron positivos a algunos de los parámetros medidos en la encuesta. Aunque también están presentes en animales negativos.
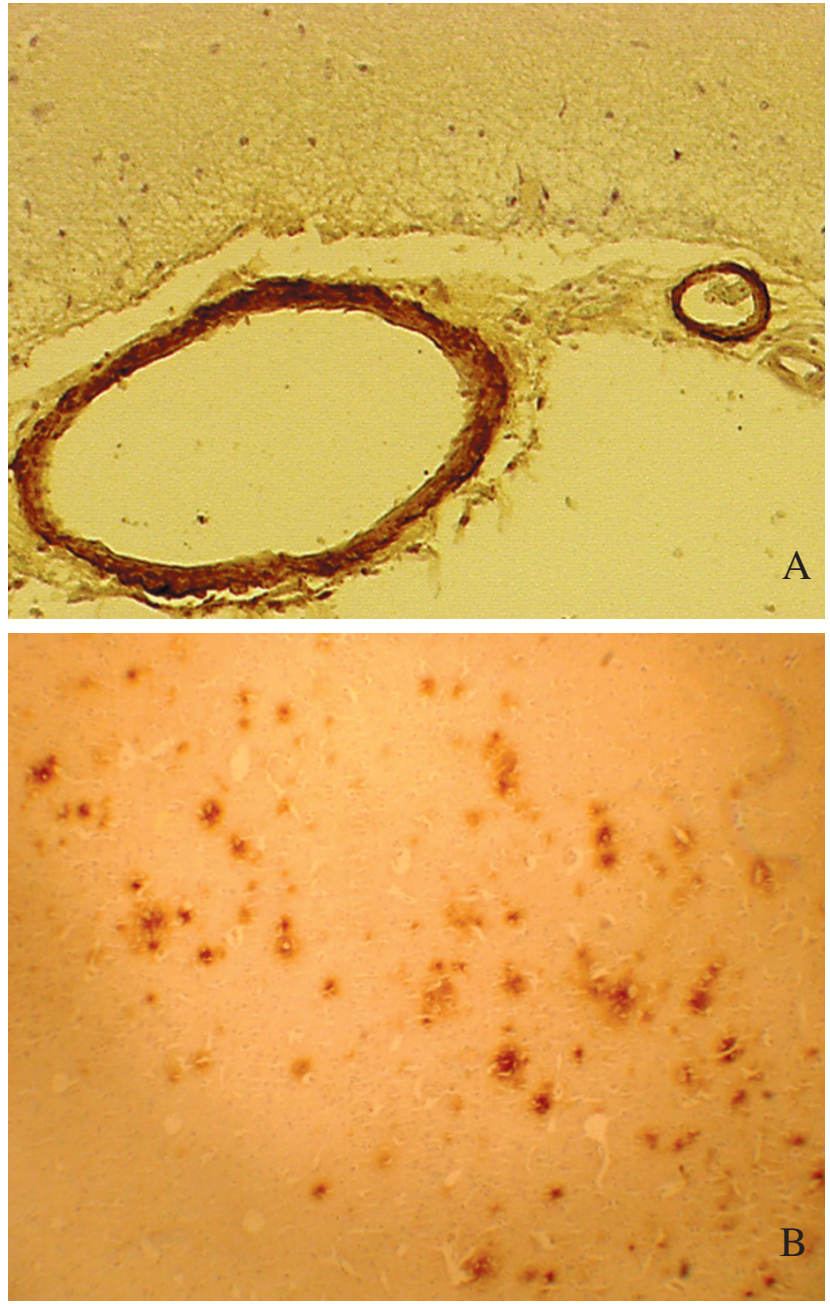

Fig. 2. A: Vasos sanguíneos leptomeníngeos en corteza frontal de canino positivo a b-amiloide (100X). B: Placas seniles difusas en corteza frontal de canino positivo a b-amiloide (400X) 
Para el anticuerpo anti proteína tau, la totalidad de las muestras resultaron inmunoreactivas, marcando células de la glia presentes en la sustancia blanca de la corteza (Fig. 3). La sustancia gris mostró sólo algunos depósitos aislados. El promedio de células positivas a proteína tau por grupos etáreos, según el análisis estadístico mostró que no hubo correlación estadísticamente significativa. Además los depósitos de proteína tau no tuvieron correlación con ninguna de las otras variables presentes en el estudio.
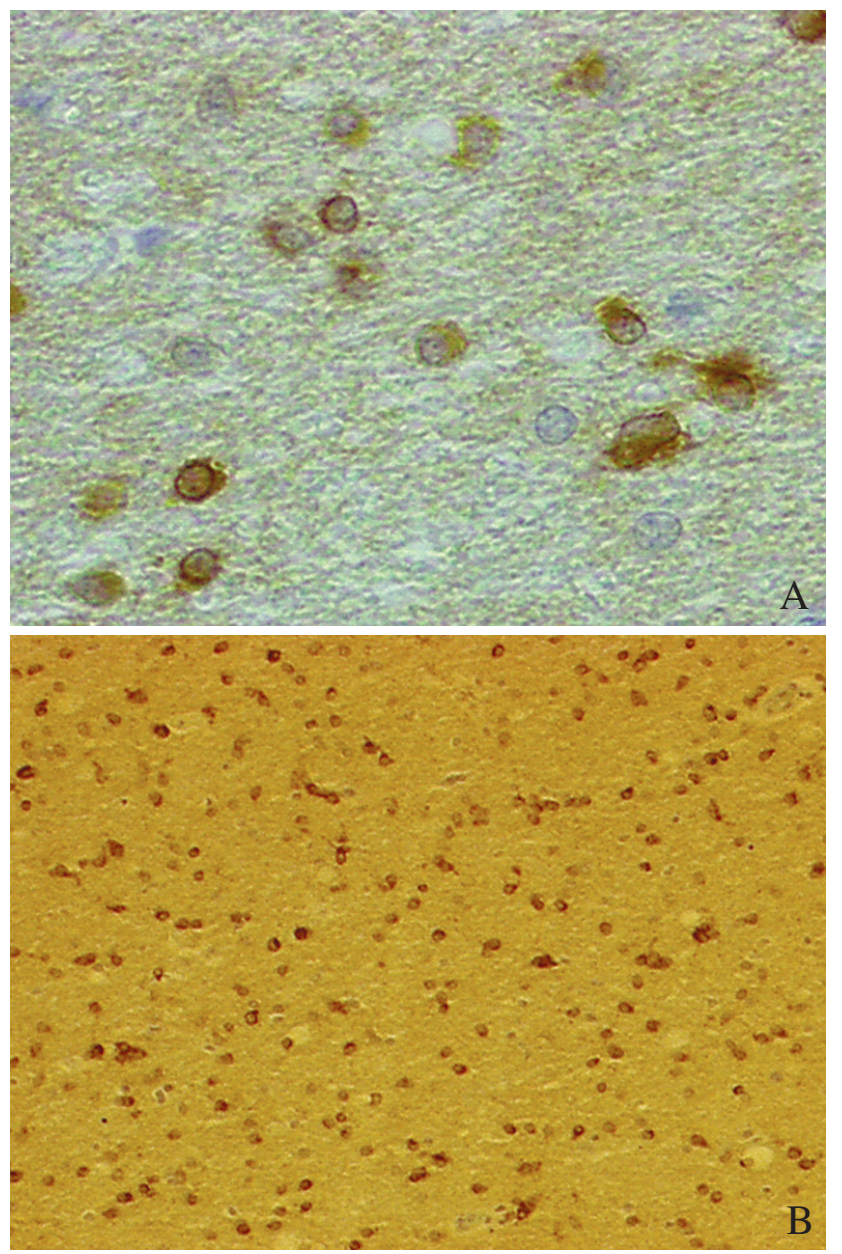

Fig. 3. A: Células de la glia (oligodendrocitos) reactivas al anticuerpo anti-proteína tau en sustancia blanca de corteza temporal canina (400X). B: lo mismo pero con aumento $100 \mathrm{X}$

Al igual que la proteína tau los depósitos de ubiquitina resultaron ser inmunorreactivos en la totalidad de las muestras. Marcó principalmente en la sustancia blanca de la corteza, zona donde se encuentran los axones (Figura 4 y 5). El promedio de gránulos y puntillado de ubiquitina por grupo etáreo presentó correlación con un valor de 0,48. Además hubo una clara tendencia a aumentar la cantidad de depósitos a medida que se incrementó la edad.
Hubo una diferencia $(\mathrm{p}<0,05)$ en la cantidad de gránulos ubiquitinados entre los grupos de 10 a 12 años y los mayores a 15 años, siendo estos últimos los que mayor cantidad presentaron. Entre los gránulos de ubiquitina y el grado de disfunción cognoscitiva hubo correlación con un valor de 0,38 .
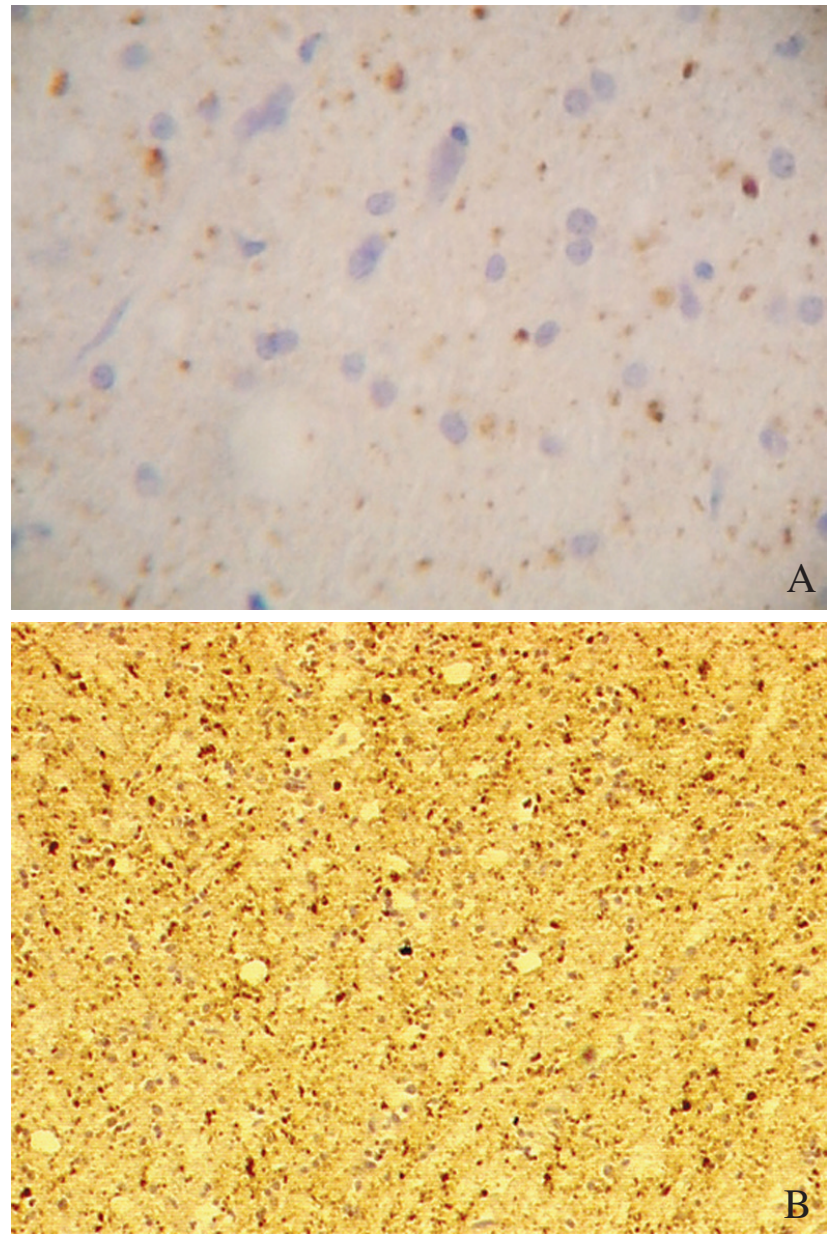

Fig. 4. Gránulos y puntillado inmunorreactivo al anticuerpo antiubiquitina en sustancia blanca de corteza temporal canina (400X).

\section{DISCUSIÓN}

Al comparar las lesiones histopatológicas encontradas en los cerebros caninos participantes en el estudio con lesiones características de la enfermedad de Alzheimer, se encontró homologación a nivel histopatológico tanto por parte de las placas de b-amiloide, los cuerpos ubiquitinados y la angiopatía amiloidea. Sin embargo a diferencia del ser humano, no tuvieron ninguna relación con el deterioro cognoscitivo que se presenta en la enfermedad, salvo para el caso de los cuerpos ubiquitinados, en donde se pudo observar que a mayor edad hubo una mayor cantidad de depó- 

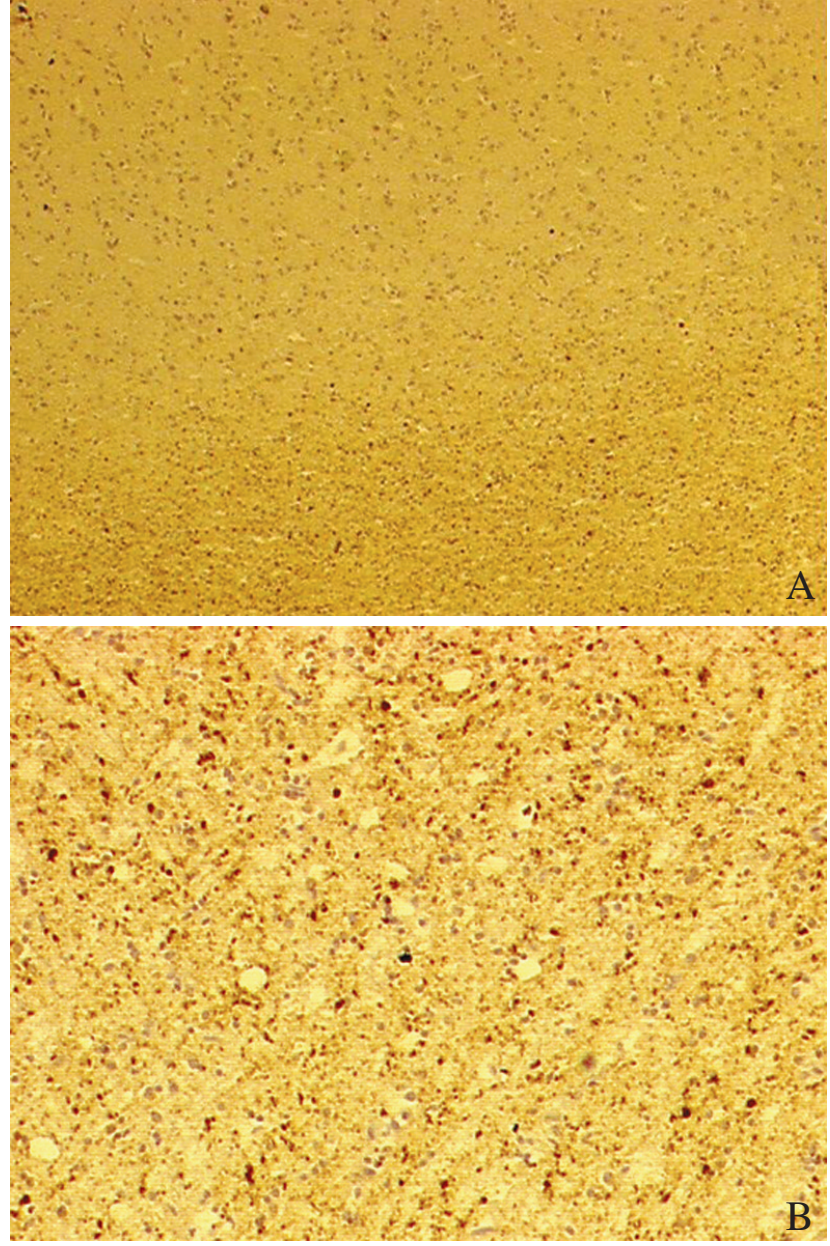

Fig. 5. A: Presencia de gránulos y puntillado inmunorreactivo al anticuerpo anti-ubiquitina en sustancia blanca de corteza temporal canina (40X). B: Gránulos y puntillado inmunorreactivo al anticuerpo anti-ubiquitina en sustancia blanca de corteza temporal canina (400X).

sitos de ubiquitina, además de presentarse un mayor deterioro cognoscitivo. Por lo tanto se puede especular que los cuerpos ubiquitinados pueden ser uno de los causantes del deterioro cognoscitivo del perro geriátrico.

Las placas de b-amiloide se encontraron en la mayoría de las muestras del estudio aunque en mayor proporción en los individuos mayores a 15 años. A pesar que la correlación entre las placas seniles y la disfunción cognoscitiva no fue estadísticamente significativa, éstas se encontraron en mayor proporción en individuos positivos a la encuesta, es decir con signos de disfunción. Pese a ésto, los depósitos también se encontraron en animales negativos a la encuesta. Por lo tanto según este estudio se constató que bamiloide es más bien un depósito relacionado con un envejecimiento normal más que patológico, a diferencia de lo que afirman otros estudios como el de Mentzel (2005b) quien aduce que el deterioro cognoscitivo se asocia a la más extensa formación de placas. Coincidente con otras investigaciones la placa presente fue la del subtipo difusa.

El anticuerpo antiproteína tau utilizado marcó células de la glia, principalmente los oligodendrocitos que se encuentran en la sustancia blanca de la corteza. No marcó ovillos neurofibrilares clásicos de la enfermedad de Alzheimer y responsables de la demencia que éstos producen en dicha patología.

No se observó correlación entre la edad y el grado de deterioro cognoscitivo sólo una tendencia a aumentar con la edad. Aunque fueron los animales mayores a 15 años los que más signos mostraron. Sin dejar de mencionar que fueron las hembras (76\%) las que mostraron mayor prevalencia al síndrome que los machos $(65 \%)$.

Se puede afirmar que la encuesta como herramienta de detección de signos asociados al Síndrome de Disfunción es de gran utilidad, siempre y cuando el dueño esté al tanto de los cambios que presentan sus mascotas, excluyendo cualquier otra patología y comportamientos típicos de la vejez que puedan inducir a errores.

Con los resultados obtenidos en esta investigación se puede reafirmar que el modelo canino está constituyendo un significado indispensable para el estudio de los procesos de neurodegeneración porque ha permitido un acercamiento a la teoría del problema desde nuevas perspectivas.

Anticuerpos de uso humano pueden ser utilizados en estudios neuropatológicos caninos, siempre y cuando se realice la estandarización con controles adecuados y pruebas correspondientes. Esto es un gran avance, ya que permitirá en el futuro seguir realizando diversos estudios, sin la necesidad de tener que usar anticuerpos especiales para caninos.

BRIONES, S. F.; CÁCERES, D. T. \& JARPA, F. M. Detection b-Amiloid, hyperphosphorylate TAU protein and ubiquitin for technique the inmunohistochemistry in brains in dogs over 10 years. Int. J. Morphol., 28(4):1255-1261, 2010.

SUMMARY: The interest in the study of the process of cerebral aging and the changes in the behaviour related to age in geriatric canine and feline has increased in the last decade (Mentzel, 2005a; Ingram \& Williams, 2010). The alterations of the canine behaviour that respond to physiopathological changes related to the age and which involve different conducts and cognitive spheres are included under the denomination of Canine Cognitive Dysfunction Syndrome (CDS) called canine Alzheimer, 
considering the cerebral and histopathologic behavioural similarities. In the present study the geriatric patients displayed the same abnormal protein deposits present in Alzheimer disease, such as senile plaques ubiquitinate bodies and the amyloid angiopathy. However they did not display the balls of neurofibrillary tangles which are characteristic of the disease. The only practical tools of evaluation in clinic for this pathology are the surveys and forms related to behavioural aspects. In addition there are post mortem exams such as those in inmunohistochemistry, that allow an effective identification of abnormal elements present in the neuropathology. In this study the monoclonal antibodies were used anti-b-amyloid, anti-protein tau and anti-ubiquitin in samples of brains in dogs over 10 years of age. The canine model constitutes an indispensable meaning for the study of the neurodegeneration processes because it has allowed an approach to the theory of the problem from a new perspective (Dimakopoulos \& Mayer, 2002).

KEY WORDS: Cognitive; Inmunohistochemistry; Amyloid; TAU protein; Ubiquitin.

\section{REFERENCIAS BIBLIOGRÁFICAS}

Alberts, B.; Bray, D.; Lewis, J.; Raff, M.; Roberts, K. \& Watson, J. Biología Molecular de la Célula. $3^{\mathrm{a}} \mathrm{ed}$. Barcelona, Ediciones Omega, 1996.

Briones, F.; Marcos, J. \& Muñoz, A. Disfunción Cognitiva en Perros Geriátricos en la Región Metropolitana, Santiago, Chile. En: XX Congreso Panamericano de Ciencias Veterinarias y $14^{\circ}$ Congreso Chileno de Medicina Veterinaria, Santiago, Chile, 2006.

Dimakopoulos, A. C. \& Mayer, R. J. Aspects of Neurodegeneration in the Canine Brain. J. Nutr., 132:1579S-82S, 2002.

Godoy, R. Ubicación y Reconocimiento de Placas Seniles, Degeneración Neurofibrilar y b-amiloide en Cerebros de Caninos Geriátricos. Trabajo de Investigación para optar al Título de Médico Veterinario. Universidad Iberoamericana de Ciencias y Tecnología, UNICIT, Santiago, Chile, 2004.

Gra, M. S; Padrón, P. N.; Llibre, R. J. J. Péptido beta amiloide, proteína Tau y enfermedad de Alzheimer. Rev. Cubana Invest. Biomed., 21:253-61, 2002.

Guimerà, A.; Gironès, X. \& Cruz-Sánchez, F. Actualización sobre la Patología de la Enfermedad de Alzheimer. Instituto de Ciencias Neurológicas y Gerontológicas. Rev. Esp. Patol., 35:21-48, 2002.
Ingram, D. \& Williams, N. Neurobiología del Síndrome de Disfunción Cognitiva en Perros, 2010. Disponible en: $\mathrm{h} \mathrm{t} \mathrm{t} \mathrm{p}:$ / / w w w. $\mathrm{m}$ e $\mathrm{v}$ e p a . c l / modules.php? name $=$ News $\&$ file $=$ article $\& \operatorname{sid}=311$.

Lozano, T. El Don de la Ubicuidad, Biología y Fisiología. Ciencia y Salud. Agosto, 1995. Disponible en: http:// servicios.laverdad.es/cienciaysalud/8_4_2.html

Mentzel, E. Síndrome de Disfunción Cognoscitiva: Aspectos Comportamentales. Memorias. V Congreso Nacional de la Asociación de Veterinarios Especializados en Animales de Compañía de Argentina (AVEACA). 8, 9 y 10 de Julio, Buenos Aires, Argentina, 2005a.

Mentzel, E. \& Juárez, M. Alteraciones del Sueño y el Animo en la Disfunción Cognoscitiva Canina. Memorias. V Congreso Nacional de la Asociación de Veterinarios Especializados en Animales de Compañía de Argentina (AVEACA). 8, 9 y 10 de Julio, Buenos Aires, Argentina, $2005 b$.

Morelli, L. \& Castaño, E. Pin y Tau en la Mira. Buscando Blancos Terapéuticos para las Enfermedades Neurodegenerativas. Ciencia Hoy, 14(83):Octubre-Noviembre, 2004. Disponible en: http:// www.cienciahoy.org.ar/ln/hoy83/proteinas.htm

Platt, S. Geriatric Brain Disease in Dogs. Memorias. V Congreso Nacional de la Asociación de Veterinarios Especializados en Animales de Compañía de Argentina (AVEACA). 8, 9 y 10 de Julio, Buenos Aires, Argentina, 2005.

Prior, R.; D’Urso, D.; Frank, R.; Prikulis, I. \& Pavlakovic, G. Loss of vessel wall viability in cerebral amyloid angiopathy. Neuroreport, 7:562-4, 1996.

Wegiel, J.; Wesniewski, H. M.; Dziewiatkowski, J.; Tarnawski, M.; Nowakowsl, J.; Dziewiatkowska, A. \& Soltysiak, Z. The origin of amyloid in cerebral vessels of aged dogs. Brain Res., 705:225-34, 1995.

Dirección para correspondencia:

Dr. Flavio Briones Silva

Médico Veterinario

Universidad Iberoamericana de Ciencias y Tecnología

Santiago

CHILE

Email: flavio@bk.ru_fbriones@gmx.net

Recibido : 30-09-2010

Aceptado: 25-10-2010 
